\title{
Rationale Behind Socially Influencing Design Choices for Health Behavior Change
}

\author{
Vasiliki Mylonopoulou ${ }^{1}$, Karin Väyrynen ${ }^{1}$, Agnis Stibe $^{2}$, Minna Isomursu ${ }^{3,1}$ \\ ${ }^{1,}$ University of Oulu, Pentti Kaiteran katu 1, 90014 Oulu, Finland \\ vasiliki.mylodoulu.fi, karin.vayrynen@oulu.fi \\ ${ }^{2}$ Paris ESLSCA Business School, 1 Rue Bougainville, 75007 Paris, France \\ a.stibe deslsca.fr \\ ${ }^{3}$ IT University of Copenhagen, Rued Langgaards Vej 7, 2300 København S, Denmark \\ miseitu.dk
}

\begin{abstract}
Persuasive technologies for health behavior change often include social influence features. Social influence in the design of persuasive technology has been described as a black box. This case study sheds light on design practices by identifying factors that affect the design of social influence features in health behavior change applications and the designers' understanding of the social influence aspects. Our findings are twofold: First, the two most positively inclined social influence features, namely cooperation and normative influence, were missing from the reviewed applications. Second, the medical condition - the persuasive technology targets - has a major influence on consideration and integration of social influence features in health behavior change applications. Our findings should be taken into account when frameworks and guidelines are created for the design of social influence features in health behavior change applications.
\end{abstract}

Keywords: Health Behavior Change, Social Influence, Design Factors.

\section{Introduction}

Different kinds of persuasive technology applications have been designed in recent years to support health behavior change facilitating social features. Different theories of persuasive technology give basis for designing among others the social features of these technologies [1-3]. However, it is unclear how designers take these theories into account and how they actually decide about the inclusion and design of social influence features. In previous research, social influence features have often been viewed as a black box [3-5], and their designs seem to be popped out without any particular explanation of why this particular feature was chosen and why it was designed in a specific way. In this paper, we address this gap through the research question: "What are the designers' rationales for the inclusion and design of social influence features in health behavior change applications?" We aim to understand and describe the designers' rationale behind their decisions on these features and the relevant design process. 


\section{Related research}

\subsection{Factors affecting the design process.}

Design process is characterized as a messy process, difficult to be described, or even taught [6,7]. The published methods and knowledge are not always used [8], and even if they are used, it is unsure if they will be perceived and used as intended [9]. More particularly, education is perceived as a way to gain knowledge, while methods are perceived as a check list or tools to help designers remember [10]. As such, the theories are used in the design as a guide, but they are not followed to the letter.

Despite the theories influencing the design through the designer's interpretations, the designer's own culture, values, and experience influence the design as well [11]. Research [12] on the design practice has described the design process as structured after the ideation phase is completed - even though there are variations on user involvement and prototype creation. The design practice has also been characterized as qualitative, subjective, and sometimes based on a gut feeling [8]. This underlines the influence of the designer's personal interpretations on the design.

According to Stolterman [13], the decisions on the design process are taken by reflecting on the theories (scientific), the practices, and the intrinsic knowledge of the designer, but als o by the client - the one served by the designer. Stolterman [6] supports that the result of the design is the result of the resources, knowledge, and involvement of the different stakeholders/clients and their desires at the particular time and place. The result of the design process (the real) is influenced by the science (the true), and the desires and wishes of the stakeholders that describe what "it ought to be" if there were no limitations (the ideal) [6]. Stolterman includes in the design process the clients and different constrains that exist in the real world to create the designed product. In addition, a focus on the stakeholders and users becomes more and more common in design thinking [14]. User-center design - where the design is based on the users, their perspectives and needs, and where in many cases the users participate in the design $[15,16]$ has been also used in healthcare for the development of applications and prototypes [17-19].

\subsection{Social influence as a black box}

In the design of behavior change applications it is common to include social features. The influence of other people on our behavior has been well established in psychology [20-22] and it has been moved to the design of technology supporting behavior change. Fogg [23] describes this technology as persuasive, because through means of persuasion and social influence the behavior change is supported. However, social influence in the design of persuasive technology oftentimes is described as a black box that needs to be opened [3-5]. Designers understand the importance to implement social influence features in behavior change technologies, but they often do not discriminate between the different social influence aspects. In fact, social influence is multifaceted, having 
such distinct sub-dimensions as social learning, social comparison, normative influence, social facilitation, cooperation, competition, and recognition [3], so it should be designed with care.

We focus on the design of social influence features (which we refer to also as social features in the text for brevity) on the health related applications and we try to understand and describe the designers' rational behind their decisions regarding these features in healthcare related applications. The aim is to understand and study the practice in order to have a better image of the design process and see if the different social aspects can be seen in the designs.

\section{$3 \quad$ Research setting and Methodology}

The research question of this study is "What are the designers' rationales for the inclusion and design of social features in health behavior change applications". In order to get in-depth insight into this question, we conducted a qualitative case study in one Spanish company that develops health behavior change applications .

The case company (Alpha) is a Spanish start-up in the area of health and IT which has close connections to academia and academic research. This increased the possibilities of the company's designers to be aware of the relevant literature/theories in addition to the commercial products, and the designers were accustomed to rationalize over their decisions also based on theory. Moreover, as it is a small company, projects were running at the same time, but in a way that the investigator could participate in and observe more than one project during the visit. The company consisted of four employees (medical advisor/PhD candidate, programmer, chief financial officer, CEO) and several co-operators. During the investigator's visit, one more $\mathrm{PhD}$ candidate was hired for the prostate cancer project. Apart from the employees, two co-operators from the local university ( $\mathrm{PhD}$ candidate, senior lecturer) were working closely with the company. The three $\mathrm{PhD}$ candidates, the programmer and the senior lecturer participated in the design of the projects described below.

Data was collected in three phases in the years 2016 and 2017. During the pre-visit period before the actual visit in Alpha (two months), two 30-minute informal-conversation interviews were conducted with the co-operators who were responsible for the smoking cessation (D) and prostate cancer (A) application, and interview notes were taken. Participant observation started in this phase, when the investigator acted as advisor (by distance) in breast cancer app (B) and was getting informed on the App D evaluation. During the visit (three months), the investigator acted as interaction designer in Alpha's projects (A, D) and additionally observed App B. She conducted four semi-structured interviews (average length: $45 \mathrm{~min}$ ) with the employees and Alpha's cooperators. Interviews were recorded and anonymized during transcription. In the post-visit period, the investigator acted as interaction designer counselor to follow the progress of the projects. The medical advisor moved to the investigator's place of work for three months, allowing the investigator to observe and participate in the design of the multiple scleroses app (C). During participant observations, notes were taken and 
transferred to the researcher's digital diary. Overall, data was collected on 10 technological applications (see Table 1). E-G were Alpha's past planned or implemented projects, and H-J were projects in the employees' past before they started to work in Alpha. Data on A-D was collected through both observation and interviews, data on E-J was collected through interviews. A-E and G-H targeted health behavior change - which is the focus of this paper. However applications related to the general behavior change within the healthcare field (F, I, J) were also included because it helped us to gain a more general understanding of designers' rationale regarding social features. It also underlined the difference between designing for patients and non-patients - users of A$\mathrm{E}, \mathrm{G}$, and $\mathrm{H}$ perceived as patients by the designers while users of $\mathrm{F}$, I, and $\mathrm{J}$ were not perceived as patients.

The data was analyzed using thematic analysis [24] and the cutting and shorting technique [25]. From the data, we extracted the rationales/justifications the designers gave for their design choices concerning social features in behavior change applications related to health issues. We identified four categories of rationales (Theories and Practices, Medical Condition, Designer's perspective, and External factors), and several sub-categories for each of them. These categories, which we will describe in more detailed in Section 4.2, arose from the data.

Table 1. Summary of health behavior change applications

\begin{tabular}{|c|c|}
\hline Application description & Social features \\
\hline $\begin{array}{l}\text { Prostate cancer app: supporting patients } \\
\text { on being physically active }(A)\end{array}$ & $\begin{array}{l}\text { Mentoring, sharing with family, community (ind. blogs \& } \\
\text { discussions), patients' paring and communication }\end{array}$ \\
\hline $\begin{array}{l}\text { Breast ca ncer app: supporting patients on } \\
\text { being physicallyactive }(B)\end{array}$ & Messaging (pre-determent), activity company \\
\hline $\begin{array}{l}\text { Multiple Sclerosis app: supporting patients } \\
\text { on managing their stamina (C) }\end{array}$ & $\begin{array}{l}\text { Feeds feature, comparison to others (on an action indi- } \\
\text { rectly connected with their condition) }\end{array}$ \\
\hline $\begin{array}{l}\text { Smoking cessation app: supporting people } \\
\text { on the smoking cessation (D) }\end{array}$ & $\begin{array}{l}\text { Messaging (one-way communication), Facebook group, } \\
\text { block feature }\end{array}$ \\
\hline $\begin{array}{l}\text { Goat disease - trivial like game: awareness } \\
\text { on goat disease }(E)\end{array}$ & $\begin{array}{l}\text { Level ranking on Facebook (the patients were grouped in } \\
\text { levels) }\end{array}$ \\
\hline $\begin{array}{l}\text { Doctor's IT knowledge - trivial like game: } \\
\text { awareness on healthcare technology (F) }\end{array}$ & Ranking on Facebook \\
\hline $\begin{array}{l}\text { Quiz \& education on male sexual health: } \\
\text { awareness and support on diagnosis }(G)\end{array}$ & Application's specifications sharing on social media \\
\hline $\begin{array}{l}\text { Diabetes app: supporting diabetic a doles- } \\
\text { cence through gamification }(\mathrm{H})\end{array}$ & Messaging/emoticons, and creation of teams \\
\hline $\begin{array}{l}\text { Medical records' completion: motivatepa- } \\
\text { tients to fill in their records though ga mifi- } \\
\text { cation elements (I) }\end{array}$ & Compareda user with the average \\
\hline $\begin{array}{l}\text { Quiz on myths in healthcare ta rgeting edu- } \\
\text { cation and awareness }(\mathrm{J})\end{array}$ & $\begin{array}{l}\text { Fa cebookga me on myths in healthcare, ranking and com- } \\
\text { parison with friends }\end{array}$ \\
\hline
\end{tabular}

\section{$4 \quad$ Results and Analysis}

In this section, we first describe our results from a micro-level view, focusing on the social features implemented in different applications as well as the designer's rationales. Then, we describe our results from a macro-level view, introducing the four 
Table 2. Reasoning around specific social features

\begin{tabular}{|c|c|c|}
\hline Social Feature & Possible Implementation & Reasoning (representative quotes) \\
\hline $\begin{array}{l}\text { Messaging/Chat } \\
\text { [SL, SF] }\end{array}$ & $\begin{array}{l}\text { Free text }(\mathrm{H}) \\
\text { Predetermined messages }(\mathrm{B}) \\
\text { One - way communication with Free text \& pre- } \\
\text { determined messages (D) }\end{array}$ & $\begin{array}{l}\text { "the first one [of the theory] -relatedness- builds with social aspects I was thinking 'hm } \\
\text { we should really have some social aspects inthis a pplication"” (H), "what if I could create } \\
\text { a tribal group of breast cancer patients that could exert their group pressure to be } \\
\text { healthy"(B), "how do I stop someone who's having a really reallybad day to get into a } \\
\text { new patient" (B) "to provide encouragement between members of the group" (D), "We } \\
\text { saw that they were happyto helpothers," (D) }\end{array}$ \\
\hline $\begin{array}{l}\text { Grouping } \\
\text { [CR] }\end{array}$ & $\begin{array}{l}\text { Gamification elements like group targets and ob- } \\
\text { ligations. }(\mathrm{H})\end{array}$ & $\begin{array}{l}\text { "So when you have this friends you all have group goal to achieve and so for exa mple if } \\
\text { you and three friends all stick to the treatment for a week, you got a bonus price and } \\
\text { then were specificchallenges that were only available if you had a group of friend that } \\
\text { were keeping you incheck and you are checking on them." (H) }\end{array}$ \\
\hline $\begin{array}{l}\text { Social Media } \\
{[S F, \text { RE] }}\end{array}$ & $\begin{array}{l}\text { Share only the application characteristics (G) } \\
\text { Share your achievements in the application (F) } \\
\text { Closed groups as a common space to connect } \\
\text { with other patients and doctor(s) (D) }\end{array}$ & $\begin{array}{l}\text { "Because we wanted to make it viral." }(G) \text {, "you have to have a player base" (E, F), "there } \\
\text { are a lot of different type of patients [...] so we did this FB group especially for them, for } \\
\text { those who are more open"(D), "they can cheer other people" (D) }\end{array}$ \\
\hline $\begin{array}{l}\text { Ranking } \\
{[C T, S C, R E]}\end{array}$ & $\begin{array}{l}\text { Ranking between friends in social media and be- } \\
\text { tween all players/medical doctors trivial(F) and } \\
\text { patients trivial( } E \text { ) } \\
\text { Ranking between friends who played the game (J) }\end{array}$ & $\begin{array}{l}\text { "we know doctors are [...] competitive [...] It was a tool to motivate them, to poke them" } \\
\text { (F), "The client wants a similar app" (E), "since they were patients, we couldn't say: 'okay, } \\
\text { you are the best patient!"” (E)"And in Facebook you would compare that to your friends } \\
\text { so it's like all of your friends have } 55 \text { correct than you have } 70 \text { you are wining and there } \\
\text { was a ranking of that." (J) }\end{array}$ \\
\hline $\begin{array}{l}\text { Comparison with } \\
\text { the AVG [SC, CT] }\end{array}$ & $\begin{array}{l}\text { Progress bar that show how much the user had } \\
\text { progressed in relation to others (I) }\end{array}$ & $\begin{array}{l}\text { "Because we wanted to see }[. . .] \text { If I can drive your behaviour with something as simple as } \\
\text { that" }(I)\end{array}$ \\
\hline $\begin{array}{l}\text { Comparison tool } \\
{[\mathrm{SC}, \mathrm{CT}]}\end{array}$ & In the ideation phase (C) & $\begin{array}{l}\text { "Social comparison between the MS patients regarding how accurate they are when they } \\
\text { estimate their energy for the activities (AB test)" (C-inv) }\end{array}$ \\
\hline $\begin{array}{l}\text { Following/Sharing } \\
\text { [SL] }\end{array}$ & In the ideation phase (C) & $\begin{array}{l}\text { "was sharing data with family in the sense that patients will not feel alone and they will } \\
\text { have the support of someone", "he wants to implement a 'following' feature" (C-inv) }\end{array}$ \\
\hline Community [SL] & In the ideation phase (A) & "with motivational experiences from PCa survivors" (A-inv) \\
\hline $\begin{array}{l}\text { Sharing } \\
{[\mathrm{SF}]}\end{array}$ & In the ideation phase (A) & $\begin{array}{l}\text { "sharing data with familyin the sense that patients will not feel alone and they will have } \\
\text { the support of someone" (A-inv) }\end{array}$ \\
\hline Mentoring [SL, SF] & In the ideation phase (A) & "one user that feels healthy can help motivating another one" (A-inv) \\
\hline
\end{tabular}

Legend: SL = Social Learning; SC = Social Comparison; SF = Social Facilitation; $\mathrm{CR}=$ Cooperation; CT = Competition; RE = Recognition 
categories of designers' rationales for social influence design choices in health behavior change applications.

\subsection{Rationales for consideration and implementation of specific social features}

In Table 2 we present the rationales behind different types of social features (micro level view). In Column 1, we present the social features that were either implemented or considered for implementation in the applications, together with a classification of which of the seven types of social features/aspects can be seen to represent (see Legend below Table 2).

In Column 2, we present the options of how that feature was (discussed to be) implemented, together with a reference to the application (A-I) within which the implementation was discussed or executed. In Column 3, we present representative interview quotations together with a reference to the application (A-I). Statements from the investigator's diary are marked with "-inv" after the application code (e.g., "A-Inv" to indicate information concerning application A was from the diary).

In general, opinions about the social aspect were controversial. They all underline the importance of the social factor on influencing people's behavior and motivation but they also reveal that some conditions are taboos and some people may be unwilling to share health information with others: "dependent on the group of the user I see social is very important in e-health." and "Well the social part in the health application is, [...] controversial part, because... not all the people want to share something [...] related to the disease". Moreover, the comments have confirmed that designers used social influence as a black box without differentiating between any of the different as pects such as social comparison, cooperation, social learning etc.: "We should really have some social aspects in this application."

The reasoning behind the different variations on features can demonstrate in a micro level the different factors influencing the designer's decisions. Messaging was included as a way to support and motivate others (see quotes at table 2 ). In one case $(\mathrm{H})$ the designer thought that the theory applied needed something social so he just chose messaging and emoticons. In anothercase (B) the messaging was predetermined text as the free text was perceived potentially harmful: "ok how do I stop someone who's having a really really bad day to get into a new patient -that is a new user for the app-and starts telling -this new user - all the bad things awaited for her in the breast cancer permeation". In a third case (D) the rationale behind the different type of messaging was influence by the interviews with patients and the designers' interpretation. Patients who wanted to quit smoking were perceived to want to support others but unwilling to have conversations. The designers decided to have one way communication i.e. to send messages to support other patients but the recipients were unable to respond: "We saw that they were happy to help others, but they didn't want to have more friends, or, they didn't want to have a new relation with someone just because they stopped smoking."

Grouping feature was implemented (I) as part of gamification of the application. The users had to create or be in groups and when that happened they received group challenges and rewards. The people in the groups were also prompted by the application to check on inactive group members through messaging. 
Social media had three variations connected to them: share, closed groups, and games. The share option was used to make the applications viral and open ( $\mathrm{G}$ and $\mathrm{J}$ ). The users could share only the details of the application in cases the disease was considered to be a taboo $(\mathrm{G})$ and they could share their achievements in a gamified app (E, F). However, there was a design difference between apps that their perceived usertarget was patients with those the target was not patients described in detail in ranking and comparisons sections.

Ranking and comparisons were used in applications that target healthy individuals or applications indirectly connected to the patients and their health such us: how complete was their electronic record in comparis on to others (I), the knowledge on a subsect (patient oriented: E, non-patient oriented: F, J), and compare an activity that is indirectly connected with the condition (C). Comparisons targeting knowledge on a disease (E) were designed with the thought that the user is a patient. The individual ranking was used in the application targeting medical doctors and their knowledge on technology (F) in order to "poke them". Later the client asked to tweak the application and target the knowledge regarding a disease (E). The designers perceived and treated the users of the application E as patients and they rationalized accordingly: "If you tell someone that 'you are the one that is at the bottom of the ranking about IT things in healthcare', that's fine, but if you tell a patient that 'you are the worst patient because you are the bottom of the ranking', that's very disappointing for the patient". The suggested design was to group the individual ranking into categories so if the user answered mostly right, then he/she is in the category "expert" or if he/she ans wered mostly wrong, he/she gets in the category "newbies" together with others. In that way, the users would not feel that they are the "worst patient". Even though the application was targeting everyone regardless of having the disease (the app was about awareness), the designers perceived the users as patients of the disease.

Not yet implemented social features are those discussed on getting implemented but not at the first stage of the application's development (A and C quotes Table 2). These features were decided to get implemented later because they were perceived as unrelated to the main focus of the applications, to the minimum viable product, or to the promised deliverable. For example, in the prostate cancer application (A) the social part was recognized and social feature suggestions (see Table 2) were made, but never implemented in the first stage, because it was perceived as unrelated to the promotion of physical activity (main target of the application as it was ordered) and the promised deliverables, and because of the lack of time and budget.

Social influence. After reviewing the collected reasoning around social influence features (Table 2), it is easy to realize that several social influence features were present (multiple times), such as social learning and social facilitation (4 times each), social comparison and competition ( 3 time each), and recognition ( 2 times). We found one sign of cooperation $(\mathrm{H})$ (tied to peer pressure). However, this was from the past experience of an employee and was not applied in any of the other applications developed in the company. This makes cooperation and normative influence completely absent from the Alpha's health behavior change applications. 


\subsection{Factors influencing the decisions}

Looking at the data in the bigger picture (macro level) we found that the decision making process of the designers implementing a social feature is complicated and influenced by more than one factor. These factors are presented in Figure 1.

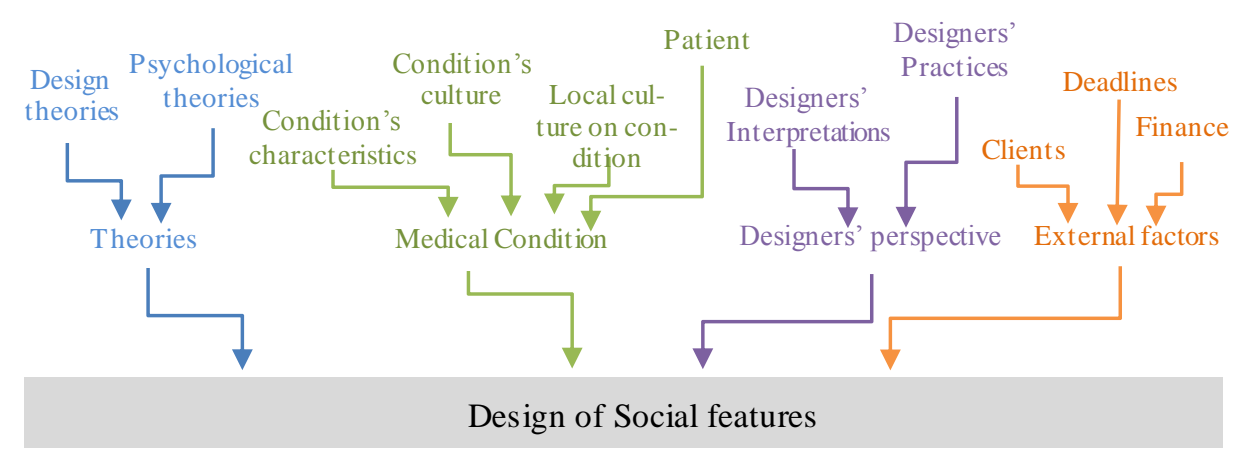

Fig. 1. Factors influencing the inclusion and design of social features

The first factor is the theories. Theories from the design field such as user-centered design have been applied in the design of the applications as methodologies that influence also the design of the social features. Users/patients were in the center of the design. Psychological theories focused on behavior change and motivation were used in the overall design of applications and also on the decisions regarding the inclusion or exclusion of a social feature: "the self-determination theory [...] says that usually we do things because of relatedness, autonomy, mastery, and purpose. And since the first one -relatedness-builds with social aspects".

The second factor is the medical condition. All the interviews show that the designers perceived the users and the users' needs first and foremost based on their condition. Namely, seeing the users through the lens of the medical condition and transforming them from users to patients. One of the sub-factors in the medical condition is the condition's characteristics. Through the interviews there were references on the stage of the condition (and how that can influence the patient), on the type of stages the condition has, or even if it has stages or it is random: "the progression of multiple scleroses it's not linear [...]. you could be completely without symptoms for 20 years, or you can [have a symptom attack] and then you [recover], or you can stay there". Another sub factor is the effect of the local culture on the condition that can have an impact on the inclusion of social features. For example, if the condition is socially unacceptable in a certain culture, then it would be tricky for the patients to share that they have the condition: "I think that's a problem - I do not know is a global problem-[...]. In prostate cancer people are men and one of the frequently disease... or commonality is eh hexual problem [...] and I think that is a problem with mentality because if I say that II am a patient with prostate cancer' people will say 'ok you are not completely man because you are sexual impotence'or something like that.'. Apart from the local culture's effect on the condition, the condition itself has a culture which may affect the inclusion of 
social features: "Now the cause of the whole tendency aimed at breast cancer women survivors, men -with breast cancer-feel completely inadequate and they are completely ashamed". Namely, this tendency in the breast cancer community can influence the design of application in inclusion of social features e.g. applications for female patients may have social features whereas applications for male patients may not. The last sub factor influence the design is the patient. The interviewees came in contact with patients, patients' families, medical professionals etc. to understand the patients who have a condition and their needs. As they base their design philosophy mainly on user-centered design, they apply different techniques to understand the patients.

The third factor influencing the design and inclusion of social features is the designer's perspective and practices. Each designer has his/her own reflection on the theories and his/her own interpretation on the data collected from patients 'relevant studies. For example, social comparis on has been perceived as gamification technique and as something secondary in an application that was to motivate prostate cancer patients to exercise: "The focus was mainly in the physical activity and secondary on selfmanagement. The social aspect get into the discussion mainly as a gamification element on the physical activity". (Investogator's diary)

The last group is the external factors from the design team, such as clients' demands, financial constraints and deadlines. The influence of the external factors can be seen in the following comments taken from the investigator's diary and the interview transcripts: "He [new PhD] also pointed out that we have to focus on physical activity because ofpractical reasons (e.g. the funder was interested in that, the [senior lecturer] insisted etc.). (Investigator's diary)" and "Interviewer: Er... How come and you implement ranking in both of them? Interviewee: Because, that's, well, er... The client wants a similar app because it was very successful with the healthcare professionals, so they said: 'ok, we want something very very similar, keep the same structure, and we introduced this little change to tweak it for patient."

Table 3 summaries our findings and presents in which of the applications A-J we found examples for the different categories of rationales. Applications which did not perceive the user as patient $(\mathrm{F}, \mathrm{I}, \mathrm{J})$ are absent in the medical condition factor.

Table 3. Categories of rationales for the design of social features

\begin{tabular}{|l|l|l|l|}
\hline Theories: & Medical condition & External factors & Designer's perspective \\
\hline - design theories: A, B, & - Medical perspective: A, B, & - Clients: C, E, F & - Personal practice : A, B, \\
C, D, F & C, D, H & - Finance: A, B & C, H, I \\
- behaviour change \& & - Condition's culture : A, B, & - Deadlines: A, C & - Personal believes /in- \\
psychological theo- & D & -Collaborators: B & terpretations: A, B, C, D, \\
ries: A, B, C, D, E & - Local culture on condi- & & E, F, G, H, J \\
& tion : A, B, G, & \\
& - Patient : A, B, C, D, E & & \\
\hline
\end{tabular}

\section{Discussion}

Theory $[3,4]$, but also practice, show that social influence features are oftentimes treated as a black box. In the previous section, we shed light on this black box by presenting 
our results regarding the designers' rationales for the inclusion and design of social influence features in health behavior change applications. We will briefly discuss our two main findings: First, the designer's rationales for including specific social influence features, and second, the role of the medical condition.

The designers quite often did refer to social support. However, such mindset is too generic for the creation and designing of specific social influence features [3]. In fact, social influence is more than just social support, which usually is implemented just as a possibility to connect and network. This finding informs that designers should be better equipped with deeper understanding of the multifaceted nature of social influence to succeed in designing effective health behavior change technologies. Moreover, the results of this study revealed that only five (social learning, social comparison, social facilitation, competition, and recognition) out of seven social influence features were present in the designers' rationales to some extent. Thus, normative influence and cooperation were absent. By their nature, the two missing features actually are some of the most positive inclined out of the seven social influence features [3].

The literature characterize the design process as messy and difficult to describe [6]. However, understanding the practice is the main step to change or support the practice by proposing a theory, framework, or method [10]. Our findings regarding three of the four categories of designers' rationales we identified in the data - Theories and Practices, Designer's perspective, and External factors - are supported by previous research. The design practice is influenced by the theories, even though they are not followed to the letter by the practice $[8,10]$. The individual characteristics of each designer (such as experience, practices, interpretations etc.) are also part of the design, as well as the constraints related to factors usually external to the design team (such as clients, funding, time limitations etc.) $[6,8,9]$. However, our study adds to previous research by identifying an additional important category in the design practices of social features in health persuasive technologies: the medical condition. In this case, the designers saw the user through the lens of a particular medical condition. They took into account the particularities of each condition (e.g., that multiple scleroses has unpredictable symptoms and the patients may feel tired most of the time). The designers took into account how the patients of this condition currently interact with each other based on the condition's culture (e.g., breast cancer patients have their communities which are mainly focused on the females). They also took into account the influence of the local culture on the condition (e.g. prostate cancer patients can be stigmatized because it is related to sexual health and males with relevant problems are consider less of males in the local culture). Finally, the designers focus on the particular patient/user through interviews and workshops, as is the case also in the user centered design [15]. The difference is that the users are perceived through the particular lens of their condition. Thus, in the designers' vocabulary and perception "the users" become "the patients" which carry with them all the pre-referred subcategories of the medical condition. 


\section{Conclusion}

In this paper we studied the designers' rationales for including social features in applications supporting health behavior change. Our contribution is twofold: first, we revealed that the designers often have a limited view on social influence that may lead to less effective designs and implementations of health behavior change technologies. This finding can help practitioners (i.e., designers of health behavior change applications) in realizing the need for acquiring more refined understanding about various social influence principles that exist and can be purposefully used for designing persuasive technologies in health domain, and possibly the need of relevant design guidelines. Second, we contribute to research by showing that the medical condition plays a significant role on the design of social features together with the scientific theories, designer's interpretation, and external factors [13]. Our results can be used to underline the importance of medical condition and its sub factors in the design practice in the field of health behavior change, and give a better understanding of the current practice for creating relevant frameworks and guidelines for better supporting the practitioners in the design of the social features [3, 4, 5, 10].

In the future more research of practice will be needed in order to inform the theory on the design practice of social influence features in persuasive technologies [3] as it is vital to know the practice before changing the relevant theory [10].

\section{$7 \quad$ Acknowledgments}

We thank all the designers contributing to this research by sharing their experiences. This publication has received funding from the European Union's Horizon 2020 research and innovation programme - Marie Sklodowska-Curie Actions grant agreement no. 676201 - CHESS - Connected Health Early Stage Researcher Support System.

\section{References}

1. Fogg BJ (2009) Creating Persuasive Technologies : An Eight-Step Design Process. Technology 91:1-6 . doi: 10.1145/1541948.1542005

2. Oinas-kukkonen H, Harjumaa M (2009) Persuasive Systems Design : Key Issues , Process Model, and System Features. Commun Assoc Inf Syst 24:485500

3. Stibe A (2015) Towards a Framework for Socially Influencing Systems: Metaanalysis of Four PLS-SEM Based Studies. In: International Conference on Persuasive Technology. Springer International Publishing

4. Oduor M, Alahäivälä T, Oinas-Kukkonen H (2014) Persuasive software design patterns for social influence. Pers Ubiquitous Comput 18:1689-1704 .

5. Mylonopoulou V, Väyrynen K, Isomursu M (2018) Designing for Behavior Change - 6 Dimensions of Social Comparison Features. In: 51st Hawaii International Conference on System Sciences. IEEE

6. Nelson HG, Stolterman E (2012) The design way : intentional change in an 
unpredictable world. The MIT Press

7. Boland RJ, Collopy F (2004) Managing as Designing. Stanford University Press

8. Zannier C, Chiasson M, Maurer F (2007) A model of design decision making based on empirical results of interviews with software designers. Inf Softw Technol 49:637-653 .

9. Gould JD, Lewis C (1985) Designing for usability: key principles and what designers think. Commun ACM 28:300-311.

10. Stolterman E (1992) How system designers think about design and methods: Some Reflections Based on an Interview Study. Scand J Inf Syst 4:

11. Razzaghi M, Jr MR (2005) The influence of the designers' own culture on the design aspects of products.... Eur Acad Des 1-15

12. Rosson MB, Maass S, Kellogg W a. (1987) Designing for designers: an analysis of design practice in the real world. Proc SIGCHI/GI Conf Hum factors Comput Syst Graph interface - CHI '87 137-142.

13. Harold G N, Stolterman E (2003) Design Judgement: Decision-Making in the Real World. Des J 6:21-31

14. Goldschmidt G, Planning T, City T, Rodgers PA (2013) The design thinking approaches of three di ff erent groups of designers based on self- reports. Des Stud 34:454-471 .

15. Norman D, Draper S (1986) User Centered System Design; New Perspectives on Human-Computer Interaction

16. Abras C, Maloney-Krichmar D, Preece J (2013) User-Centered Design. 154 .

17. Monteiro-guerra F, Rivera-Romero O, Mylonopoulou V, et al (2017) The Design of a Mobile App for Promotion of Physical Activity and SelfManagement in Prostate Cancer Survivors : Personas, Feature Ideation and Low- Fidelity Prototyping. In: 30TH IEEE International Symposium on Computer-Based Medical Systems. IEEE, Thessaloniki

18. Johnson CM, Johnson TR, Zhang J (2005) A user-centered framework for redesigning health care interfaces. J Biomed Inform 38:75-87 .

19. Clemensen J, Larsen SB, Kyng M, Kirkevold M (2007) Participatory Design in Health Sciences: Using Cooperative Experimental Methods in Developing Health Services and Computer Technology. Qual Health Res 17:122-130 .

20. Arons on E (2003) The social animal. Macmillan

21. Festinger (1954) A Theory of Social Comparison Processes. Hum Relations 7:117-140 .

22. Buunk B, Gibbons FX (2016) Health, coping, and well-being : perspectives from social comparis on theory. Routledge

23. Fogg BJ (2003) Persuasive Technology: Using Computers to Change What We Think and Do

24. Guest G, MacQueen KM, Namey EE (2012) Applied thematic analysis. Sage Publications

25. Lincoln YS, Guba EG (1985) Naturalistic inquiry. Sage Publications 